\title{
Jäger und Gejagte - wie sich Pilze gegen räuberische Amöben wehren
}

\author{
IULIIA FERLING, SILVIA RADOSA, FALK HILLMANN \\ LEIBNIZ-INSTITUT FÜR NATURSTOFF-FORSCHUNG UND INFEKTIONSBIOLOGIE - \\ HANS-KNÖLL-INSTITUT, JENA
}

\section{What seems obvious for most free-living bacteria, also appeals for yeast and filamentous fungi: their natural reservoirs include a variety of micropredators, such as members of the Amoebozoa kingdom. Not only do they share a predatory lifestyle, but their amoeboid motility and way of ingesting living microbial food reveals several similarities to innate immune cells. Understanding how fungi have learned to cope with such environmental phagocytes will shed new light on the evolutionary driving forces of fungal diversity and virulence.}

DOI: $10.1007 / \mathrm{s} 12268-021-1617-1$

(C) Die Autorinnen und Autoren 2021

Es gibt wohl kein terrestrisches Ökosystem ohne Pilze. Als Zersetzer organischen Materials leben sie häufig in mutualistischen oder parasitären Beziehungen zu anderen Organismen. Als Krankheitserreger treten Pilze nicht nur bei landwirtschaftlichen Nutzpflanzen in Erscheinung, sondern sorgen zunehmend für klinische Herausforderungen. Gewebsinvasive oder systemische Pilzinfektionen sind zwar im Vergleich zu bakteriellen Infektionen seltener, dafür aber häufig nur schwer diagnostizier- und behandelbar. Die Erreger von Mykosen sind divers: Hefen der Gattung Candida leben als Kommensalen auf
Schleimhäuten warmblütiger Tiere und Menschen, während Cryptococcus neoformans von Bäumen, aus Böden oder Taubenkot isoliert wurde. Pneumocystis jirovecii ist dagegen ein obligater, intrazellulärer Parasit menschlicher Zellen, dessen Kultivierung im Labor bis heute nicht möglich ist. Unter den filamentösen Pilzen tritt Aspergillus fumigatus am häufigsten klinisch in Erscheinung. Gleichzeitig ist er durch die Verbreitung über Sporen ubiquitär verbreitet.

\section{Schimmelpilze - wehrhafte} Saprophyten mit Infektionspotenzial Das Wirtspektrum von A. fumigatus ist alles andere als spezifisch und umfasst neben immunsupprimierten Menschen auch Reptilien und Vögel. In der Regel gehen die gewebsinvasiven Hyphen in der Lunge auf wenige eingeatmete Sporen (Konidien) zurück, die den Angriff phagozytierender Immunzellen überlebt haben. Diese Resistenz gegenüber Makrophagen war Motivation für zahlreiche Studien zur Oberflächenstruktur der Sporen. Hier liegen die besonders immu-

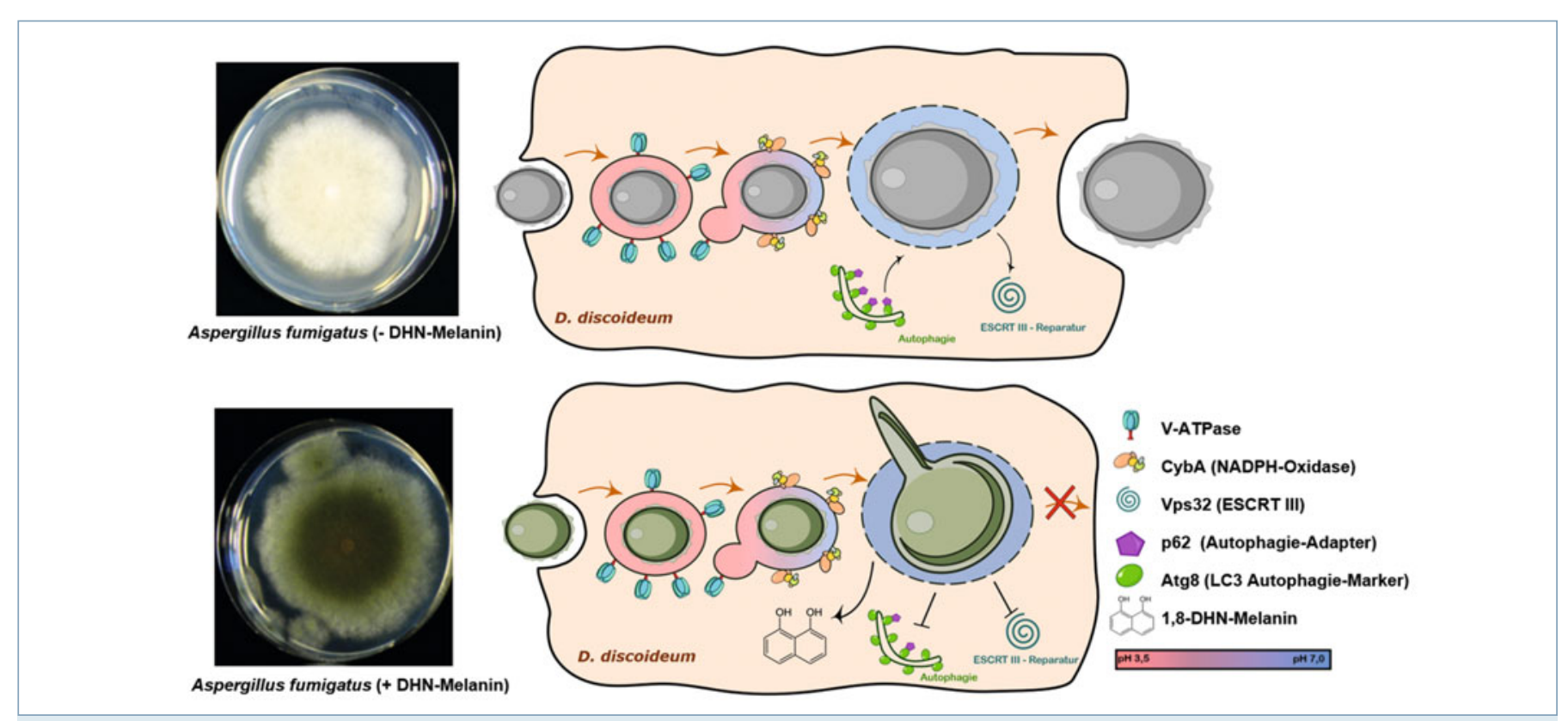

$\Delta$ Abb. 1: Phagozytische Prozessierung von Aspergillus fumigatus-Konidien in der Amöbe Dictyostelium discoideum. Im Gegensatz zu unpigmentierten Pilzsporen (oben, weiße Kolonie), verzögern melanisierte Sporen (unten, grüne Kolonie) die intrazelluläre Prozessierung und anschließende Exozytose. Schwellung und Keimung beschädigen die Membran des Phagolysosoms, wobei DHN-Melanin die Rekrutierung der ESCRT-Reparaturmaschinerie hemmt. 
nogenen Kohlenhydratpolymere der Zellwände wie $\beta-1,3-G l u c a n$ und Chitin in der ruhenden Spore allerdings unter einem hydrophoben Mantel aus Proteinen und dem amorphen Pigment 1,8-DHN-Melanin verborgen. Letzteres verleiht den Pilzsporen ihre charakteristischen grau-grüne Farbe (Abb. 1). Wie andere Pilze verfügt $A$. fumigatus dazu über ein Arsenal von Naturstoffen, wovon einige immunsuppressive Wirkung entfalten.

Welche Rolle spielen diese Virulenzfaktoren im natürlichen Habitat? Für pathogene Bakterien, die menschliche Wirtszellen aktiv infizieren, wie z. B. Legionella und Listeria, ist bereits bekannt, dass sich ihre Virulenzfaktoren auch gegen Amöben als allgegenwärtige Mikroprädatoren richten können. Auch Hefen und filamentöse Pilze unterliegen einem Fraßdruck durch Amöben. So fand man bereits in den 1970er- und frühen 1980er-Jahren immer wieder ausgehöhlte Sporen und leere Hyphen. Die Pilze waren Opfer verschiedener hochkomplexer amöboider Einzeller geworden, zumeist aus der Gruppe der Cercozoa (Vampyrella sp.) oder Amoebozoa (Gephyramoeba, Saccamoeba, Thecamoeba) [1].

\section{Amöben und Pilze - zwischen Modell und natürlichen Interaktionspartnern}

Dass Amöben und Pilze interagieren, entdeckte Aldo Castellani schon vor fast 100 Jahren, als Acanthamöben eine CryptococcusKultur kontaminierten [2]. Acanthamoeba castellanii wurde später das erste AmöbenPhagozytose-Modell für Pilze. Bei der Hefe Cryptococcus neoformans verläuft eine Infektion fast nur bei AIDS-Patienten systemisch und lebensbedrohlich. Die Hefezellen werden in der Regel aus der Umwelt aufgenommen und können von der Lunge ausgehend bis in Gehirn gelangen, wo sie fatale Entzündungen auslösen können. Vermutlich transportieren Makrophagen sie dorthin, die von den Hefen infiziert werden. Zu bestimmten Zeitpunkten können die Hefen ihr trojanisches Pferd wieder verlassen, entweder durch Lyse der Wirtszelle oder eine plötzliche, nicht lytische Exozytose, die die Entdecker auf den Namen „Vomozytose“ (engl. vomit) brachte [3].

Interessanterweise laufen diese Prozesse nach nahezu identischem Muster auch im beliebten zellbiologischen Modell Dictyostelium discoideum ab. Zudem wird die Hefe nach längerer Exposition und einem sich wiederholenden Transfer von Cryptococcus durch die Amöben resistenter gegenüber Makrophagen [4].

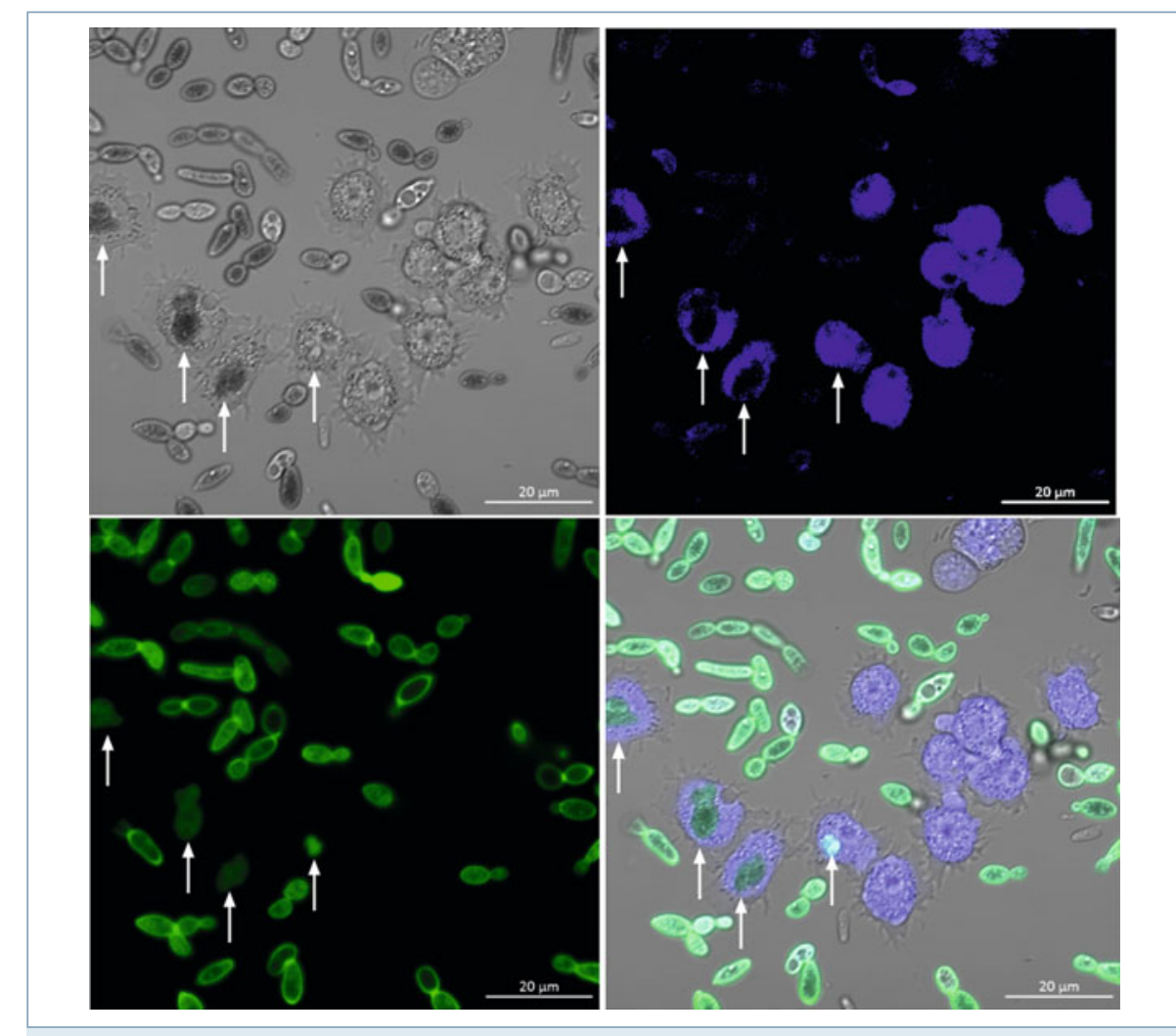

$\triangle$ Abb. 2: Protostelium aurantium (blau) frisst Hefezellen von Rhodotorula mucilaginosa (grün). Die mikroskopische Aufnahme zeigt grün fluoreszierende Zellen der Hefe $R$. mucilaginosa (Färbung mit Fluorescein-Isothiocyanat) und $P$. aurantium (gefärbt mit CMCA, CellTrackerTM Blue). Weiße Pfeile kennzeichnen Amöben, die bereits Hefezellen phagozytiert haben.

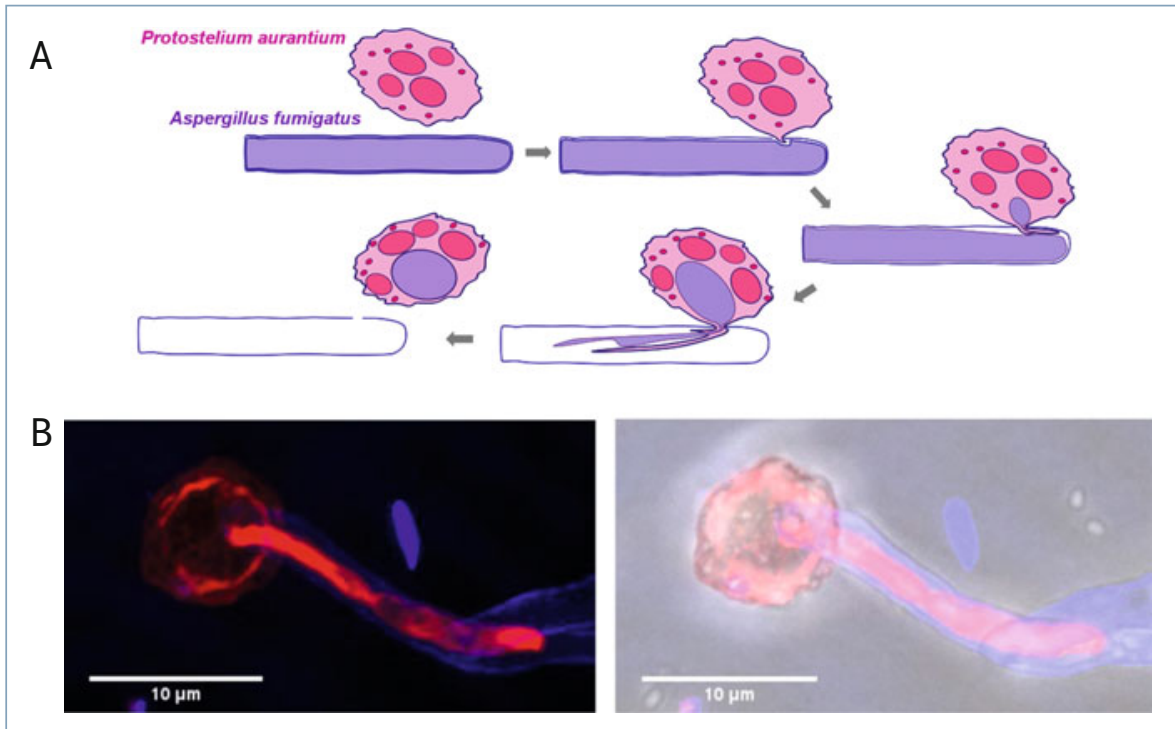

$\Delta$ Abb. 3: Ruphozytose („Aussaugen“) von Protostelium aurantium an Hyphen des Schimmelpilzes Aspergillus fumigatus. A, Schema der Interaktion. Die Amöbe attackiert Hyphen zunächst an der Spitze und öffnet die Zellwand, sodass eine flexible, fingerartige Struktur in die Hyphe eingeführt und anschließend das Cytoplasma resorbiert wird. Zurück bleibt die leere Zellwandhülle. B, Mikroskopische Aufnahme der Ruphozytose (blau: Chitin in der Zellwand von A. fumigatus; rot: F-Actin von $P$. aurantium)

Die Acanthamoeba- und DictyosteliumModelle lassen sich auch auf die Sporen von A. fumigatus anwenden und zeigen, dass die
Amöben die Sporen zwar aufnehmen können, diese dann aber innerhalb der Zelle ihre Keimung einleiten und somit die Wirtszelle 
von innen lysieren [5]. Ein ähnliches Bild wurde bei Makrophagen beobachtet. Mittlerweile ist bekannt, dass DHN-Melanin oder dessen Abbauprodukte sich während des Schwellens von der Sporenoberfläche lösen. Diese würde normalerweise zu einer Erkennung des $\beta$-1,3-Glucans der Zellwand führen, und die Makrophagen würden ähnlich wie bei einer Autophagie den Verdau der Spore einleiten [6]. Durch DHN-Melanin oder dessen Fragmente wird jedoch die Verarbeitung der phagozytierten Spore in Makrophagen deutlich verzögert, u. a. werden Flöße aus Lipiden, die lipid rafts, in der Phospholipidmembran des Phagolysosoms nicht mehr korrekt ausgebildet, und somit können wesentliche Proteine der Wirtszelle nicht korrekt positioniert werden [7].

Auch bei der Interaktion mit Amöben spielt das DHN-Melanin eine entscheidende Rolle für den Ausgang der Interaktion. Während unpigmentierte Sporen von D. discoideum innerhalb von einer Stunde wieder ausgeschieden werden, verbleiben Sporen mit einer Melaninschicht bis zu zwei Stunden oder länger innerhalb der Wirtszelle. In diesem Fall verzögert das Pigment ebenfalls die Prozessierung des Amöben-Phagolysosoms. Aufgrund der längeren Verweilzeit stellten wir zudem vermehrte Schäden an der phagolysosomalen Membran fest (Abb. 1), wobei eindeutig erkennbar ist, dass die notwendigen Reparaturproteine als Teil der endosomal sorting complex required for transport machinery (ESCRT) nicht am Phagolysosom lokalisiert sind [8]. Die Schäden an der Membran führen dabei möglicherweise zum Einstrom von Cytoplasma, was wiederum die Keimung des Pilzes beschleunigen könnte.

\section{Protostelium aurantium: fungivore Amöbe mit breitem Speiseplan}

Handelt es sich bei der Abwehr von Amöben lediglich um Analogien zu Vorgängen mit Makrophagen, oder können so widerstandsfähige Pilze wie A. fumigatus tatsächlich Fressfeinden aus der Gruppe der Amoebozoa ausgesetzt sein? Tatsächlich gibt es sogar unter den Verwandten der Dictyosteliden pilzfressende Vertreter. Hierbei handelt es sich um viele Arten der Protosteliden, Kosmopoliten die häufig von Blattoberflächen isoliert werden können. Unsere Charakterisierung von Protostelium aurantium verweist auf eine strikt fungivore Lebensweise [9]. Während P. aurantium in Gegenwart von Bakterien nicht wächst, konsumiert sie ein weites Spektrum von Hefen, wobei Candida parapsilosis oder Rhodotorula mucilaginosa favorisierte Nahrungsquellen sind. Überraschend ist zudem, dass sich $P$. aurantium bei der Nahrungssuche nicht nur auf die Phagozytose „einfacher“ Hefen beschränkt (Abb. 2). P. aurantium phagozytiert und tötet auch Sporen von A. fumigatus, allerdings nur, wenn deren melaninhaltige Hülle entweder durch Schwellung oder Mutation verloren gegangen ist [8]. Auch ganze Hyphen werden attackiert. In einem Vorgang, den wir als „Rupho-

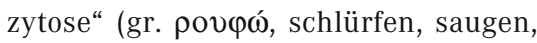
Abb. 3) bezeichnen, öffnet die Amöbe die Hyphe, dringt durch Konzentration ihrer Aktinfilamente in das Innere der Hyphe ein und verlässt sie wieder, nachdem das Cytoplasma resorbiert wurde [10]. Ähnliche Vorgänge wurden auch schon bei anderen Protozoen beobachtet, die sich von filamentösen Algen ernähren.

Es spricht also vieles dafür, dass auch die Sporen und Hyphen der meisten filamentösen Pilze in ihren Habitaten solchen Mikroprädatoren seit Millionen von Jahren ausgesetzt sind und den Jägern eine Fülle von Sekundärmetaboliten entgegensetzen. Einige davon entfalten ihre Wirkung wohl auch gegen phagozytische Immunzellen, was für diese Pilze wiederum die Chance erhöht, einen Wirt zu kolonisieren. Derzeitige Forschungen sollen zeigen, ob die molekularen Faktoren, mit denen Amöben Pilze derart spezifisch erkennen und abtöten, auch diagnostische oder therapeutische Verwendung finden könnten.

\section{Literatur}

[1] Old KM, Darbyshire JF (1978) Soil fungi as food for giant amoebae. Soil Biol Biochem 10: 93-100

[2] Castellani A (1930) An amoeba found in culture of yeast: Preliminary note. J Trop Med Hyg 33: 16 [3] Ma H, Croudace JE, Lammas DA, May RC (2006) Expulsion of live pathogenic yeast by macrophages. Curr Biol 16: 2156-2160

[4] Steenbergen JN, Nosanchuk JD, Malliaris SD,

Casadevall A (2003) Cryptococcus neoformans virulence is enhanced after growth in the genetically malleable host Dictyostelium discoideum. Infect Immun 71: 48624872

[5] Hillmann F, Novohradská S, Mattern DJ et al. (2015) Virulence determinants of the human pathogenic fungus Aspergillus fumigatus protect against soil amoeba predation. Environ Microbiol 17: 2858-2869

[6] Akoumianaki T, Kyrmizi I, Valsecchi I et al. (2016) Aspergillus cell wall melanin blocks LC3-associated phagocytosis to promote pathogenicity. Cell Host \& Microbe 19: 79-90

[7] Schmidt F, Thywißen A, Goldmann M et al. (2020)

Flotillin-dependent membrane microdomains are
Hier steht eine Anzeige.

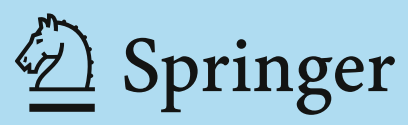


required for functional phagolysosomes against fungal infections. Cell Rep 32: 108017

[8] Ferling I, Dunn JD, Ferling A et al (2020) Conidial melanin of the human-pathogenic fungus Aspergillus fumigatus disrupts cell autonomous defenses in amoebae. mBio 11: 00862-00820

[9] Hillmann F, Forbes G, Novohradská S et al. (2018) Multiple roots of fruiting body formation in amoebozoa. Genome Biol Evol 10: 591-606

[10] Radosa S, Ferling I, Sprague JL et al. (2019) The different morphologies of yeast and filamentous fungi trigger distinct killing and feeding mechanisms in a fungivorous amoeba. Environ Microbiol 21: 1809-1820

Funding note: Open Access funding enabled and organized by Projekt DEAL. Open Access: Dieser Artikel wird unter der Creative Commons Namensnennun 4.0 International Lizenz verơffentlicht, welche die Nutzung, Vervielfältigung, Bearbeitung, Verbreitung und Wiedergabe in jeglichem Medium und Form erlaubt, sofern Sie den/die ursprünglichen Autor(en) und die Quell

ordnungsgemäß nennen, einen Link zur Creative Commons Lizenz beifügen und

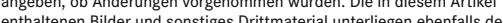
genannten Creative Commons Lizenz, sofern sich aus ser Abbilds der genannten Creative Commons Lizenz, sofern sich aus der Abbildungslegend genannten Creative Commons Lizenz steht und die betreffende Handlung nicht nach gesetzlichen Vorschriften erlaubt ist, ist für die oben aufgeführten Weiterverwendungen des Materials die Einwilligung des jeweiligen Rechteinhabers einzuholen. Weitere Details zur Lizenz entnehmen Sie bitte der Lizenzinformation auf http://creativecommons.org/licenses/by/4.0/deed.de.

\section{Korrespondenzadresse:}

Dr. Falk Hillmann

Leibniz-Institut für Naturstoff-Forschung und Infektionsbiologie e. V. - Hans-Knöll-Institut (HKI) Beutenbergstraße 11 a

D-07745 Jena

falk.hillmann@leibniz-hki.de

\section{AUTORINNEN UND AUTOREN}

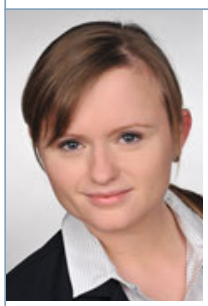

luliia Ferling

2003-2009 Studium der Ökobiotechnologie an der National Aviation University Kiew,

Ukraine. 2010-2014 Studium der Agrobiotechnologie an der Universität Gießen. 2015-2019 Doktorandin in der Gruppe von Dr. F. Hillmann am Leibniz-Institut für Naturstoff-Forschung und Infektionsbiologie e. V. - Hans-Knöll-Institut (HKI), Jena.

Seit 2021 Postdoktorandin bei Prof. Dr. S. Grinstein am Hospital for Sick Children, Toronto, Kanada.

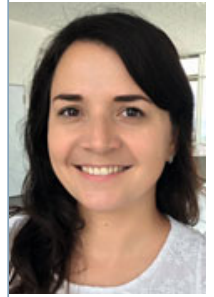

\section{Silvia Radosa}

2005-2010 Bachelor- und Masterstudium der Biologie bzw. Mikrobiologie an der Comenius-Universität Bratislava, Slowakei. 2014-2019 Doktorandin und seit 2020 Postdoktorandin in der Gruppe von Dr. F. Hillmann am Leibniz-Institut für NaturstoffForschung und Infektionsbiologie e. V. - Hans-Knöll-Institut (HKI), Jena.

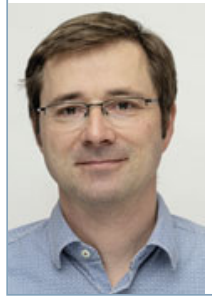

\section{Falk Hillmann}

1999-2009 Biologiestudium und Promotion in Mikrobiologie an der Universität Rostock bei Prof. Dr. H. Bahl. 2009-2011 Postdoktorand am Institut Pasteur bei Prof. Dr. A. P. Pugsley, Paris, Frankreich. 2011-2015 Wissenschaftlicher Mitarbeiter am Leibniz-Institut für Naturstoff-Forschung und Infektionsbiologie e. V. - Hans-KnöllInstitut (HKI) bei Prof. Dr. A. Brakhage. Seit 2016 Leiter der unabhängigen Nachwuchsgruppe Evolution Mikrobieller Interaktionen.

\section{Hier steht eine Anzeige.}

\section{照 Springer}

